\title{
An accurate two-dimensional theory for deformation and stress analyses of functionally graded thick plates
}

\author{
D. K. Jha $\cdot$ Tarun Kant $\cdot$ R. K. Singh
}

Received: 28 January 2013/ Accepted: 15 June 2014/Published online: 27 June 2014

(c) The Author(s) 2014. This article is published with open access at Springerlink.com

\begin{abstract}
A displacement-based, higher order shear and normal deformations theory (HOSNT) is used to analyse the thick functionally graded (FG) plates in which mechanical properties are changing smoothly with the plate's thickness coordinate. A more realistic parabolic distribution of transverse shear strains through thickness of plate is ensured in the presented formulation. The influence of transverse normal strain on bending responses of FG plates is examined in this study. Functionally graded materials (FGMs), although heterogeneous, are idealized as continua with their mechanical properties changing smoothly with respect to spatial coordinates. The material properties of FG plates are assumed to be varying through thickness of plate in a continuous manner. Poisson's ratio of FG plates is assumed to be constant, but their Young's modulii are considered to vary continuously in thickness direction according to the volume fraction of its constituents which is mathematically modelled as an exponential function. The governing equations of equilibrium for static analysis of FG plates are obtained using principle of minimisation of potential energy (PMPE) employing HOSNT. Navier solution method is used to solve the governing differential equations of
\end{abstract}

D. K. Jha $(\bowtie)$

Civil Engineering Division (CED), Bhabha Atomic Research

Centre (BARC), Department of Atomic Energy, Trombay,

Mumbai 400 085, India

e-mail: dkjha@barc.gov.in

T. Kant

Department of Civil Engineering, Indian Institute of Technology

(IIT) Bombay, Powai, Mumbai 400 076, India

R. K. Singh

Reactor Safety Division (RSD), Bhabha Atomic Research Centre (BARC), Department of Atomic Energy, Trombay,

Mumbai 400 085, India equilibrium. It is thought that the presented results would be a reference for other researchers to compare their results.

Keywords Higher order shear and normal deformation theory · Functionally graded plates · Material grading index $\cdot$ Navier solution

\section{Introduction}

Functionally graded materials (FGMs) are the advanced materials of the family of engineering composites made of two or more constituent phases with a continuous and smoothly varying composition (Koizumi 1997). These advanced materials with engineered gradients of composition, structure and/or specific properties in the preferred direction/orientation are superior to homogeneous material composed of similar constituents. These materials possess numerous advantages that make them appropriate in potential applications including the potential reduction of in-plane and through-the thickness transverse stresses, improved thermal properties, high toughness, etc. FGMs consisting of metallic and ceramic components are well known to enhance the properties of thermal-barrier systems, because cracking or de-lamination, which are often observed in conventional multi-layer systems are avoided due to the smooth transition between the properties of the components. By varying percentage contents of volume fractions of two or more materials spatially, FGMs can be formed which will have desired property gradation in spatial directions. The mechanical properties such as Young's modulus of elasticity, Poisson's ratio, shear modulus of elasticity, material density, etc. vary smoothly and continuously in preferred directions in FGMs because of continuous changes in the composition, microstructure, 
porosity, etc. These materials are gaining wide applications in various branches of engineering and technology with a view to make proper use of potential properties of the available materials in the best possible way. FGMs can be employed in thin-walled members, like plates and shells which are used in nuclear reactor vessels, turbines and other machine parts, which are prone to failure from buckling, large amplitude deflections, or excessive stresses induced by thermal or combined thermo-mechanical loading. Functionally graded (FG) coatings on these structural elements may help in reducing the failures.

An excellent introduction to the fundamentals of FGMs has been provided by Suresh and Mortensen (1998) presenting a very detailed literature review in FGM technology. Since then, numerous investigators have attempted a variety of analytical and numerical methods for studying the mechanical, thermal and dynamic responses of structures made of FGMs. Birman and Byrd (2007) have documented an exhaustive literature review of developments in FGM research addressing the characterization, modelling and analysis of FGMs. The exact three-dimensional (3D) elasticity solutions of simply supported laminated plates have been provided by Pagano $(1969,1970)$, Srinivas and Rao (1970) and Srinivas et al. (1970). Their benchmark solutions have proved to be very useful in assessing two-dimensional (2D) approximate plate theories by various researchers (Kant 1982; Pandya and Kant 1988; Kant and Manjunatha 1994; Reddy 1997; Kant and Swaminathan 2002). These investigations are valid for laminated plates and shells, where the material properties are piecewise constant, but not applicable for finding solutions of plate problems with continuous inhomogeneity of material properties such as FGMs. Mian and Spencer (1998) presented a set of exact solutions of the 3D elasticity equations for traction-free rectangular and circular isotropic FG plates from the corresponding planar problems. They derived an exact solution of 3D elasticity equations for isotropic linearly elastic, inhomogeneous materials actually generalised from the solutions for stretching and bending of symmetrically inhomogeneous plates. The investigators showed that the exact 3D solutions are generated by 2D solutions of the thin-plate equations for a homogeneous plate. They actually developed a procedure for constructing the exact solutions of the linear elasticity equations of the plates in an inhomogeneous isotropic material assuming the elastic modulii depend in any specified manner on a specific direction. Vel and Batra (2002) presented the exact 3D elasticity solutions of the static thermo-mechanical problems of a simply supported rectangular thin and thick FG plates. The thermal and mechanical loads were imposed on the top and bottom surfaces of the plate either individually or simultaneously. 3D analytical solutions for FG plates are very useful since they provide benchmark results to assess the accuracy of various $2 \mathrm{D}$ plate theories and finite element formulations, but their solution methods involve mathematical complexities and are very difficult and tedious to solve. Ferreira et al. $(2005,2007)$ have investigated the static deformations of simply supported FG plates using TSDT and a meshless method considering the collocation multi-quadric radial basis functions (RBF). The effective material properties were calculated using the rule of mixtures and the Mori-Tanaka scheme. Nguyen et al. (2007, 2008) have generalised the Whitney's approach for deriving shear correction factors for FG plates using the first-order shear deformation theory (FOST) for modelling the structures made of FGMs. The material properties were assumed to be isotropic at each point and vary through the thickness according to a power law distribution. The improved shear stiffnesses and shear correction coefficients for the FG plate models using FOST were presented in their studies. The obtained results show that the shear correction factor is not the same as the one of the homogeneous FOST models and is a function of the ratio between elastic modulii of constituents and of the distribution of materials through the models. For a complete reference of recent research studies on the static, vibration and stability analyses of FG plates, the readers may refer to the critical review on the subject matter by Jha et al. (2013).

In most of the 2D theories developed to predict the global responses of FG plates, only the transverse shear deformation effect has been considered and very few theories consider the effect of both transverse shear and transverse normal deformations effect. In most of the 2D shear deformation theories developed till date, the validation and accuracy of the global responses of FG plates are done by comparing the results with $3 \mathrm{D}$ elasticity solutions. Very limited studies are reported on comparison of the accuracy with analytically predicted global responses of FG plates using various higher order theories. It is necessary to develop new methodologies for the analysis and design of structural components, viz., beams, plates and shells made of these advanced materials with reasonably high accuracy and computational efforts. This is definitely essential in view of the potential applications of FGMs in various fields such as special nuclear components (in fusion reactors), spacecraft structural members, ceramics, composites and high-temperature thermal barrier coatings, etc. Due to the special properties exhibited by the FGMs, such as high degree of anisotropy, higher load carrying capacity due to membrane-flexure coupling for preferential structural performance, the method of analysis based on classical plate theory (CPT) which neglects the effect of out-ofplane (transverse) stresses/strains become inadequate. FOST assumes constant states of transverse shear stresses and requires the use of shear correction coefficients to 
Fig. 1 Geometry of sinusoidally loaded FG plate with positive set of reference axes and its displacement components (for displacement model 'HOSNT11')

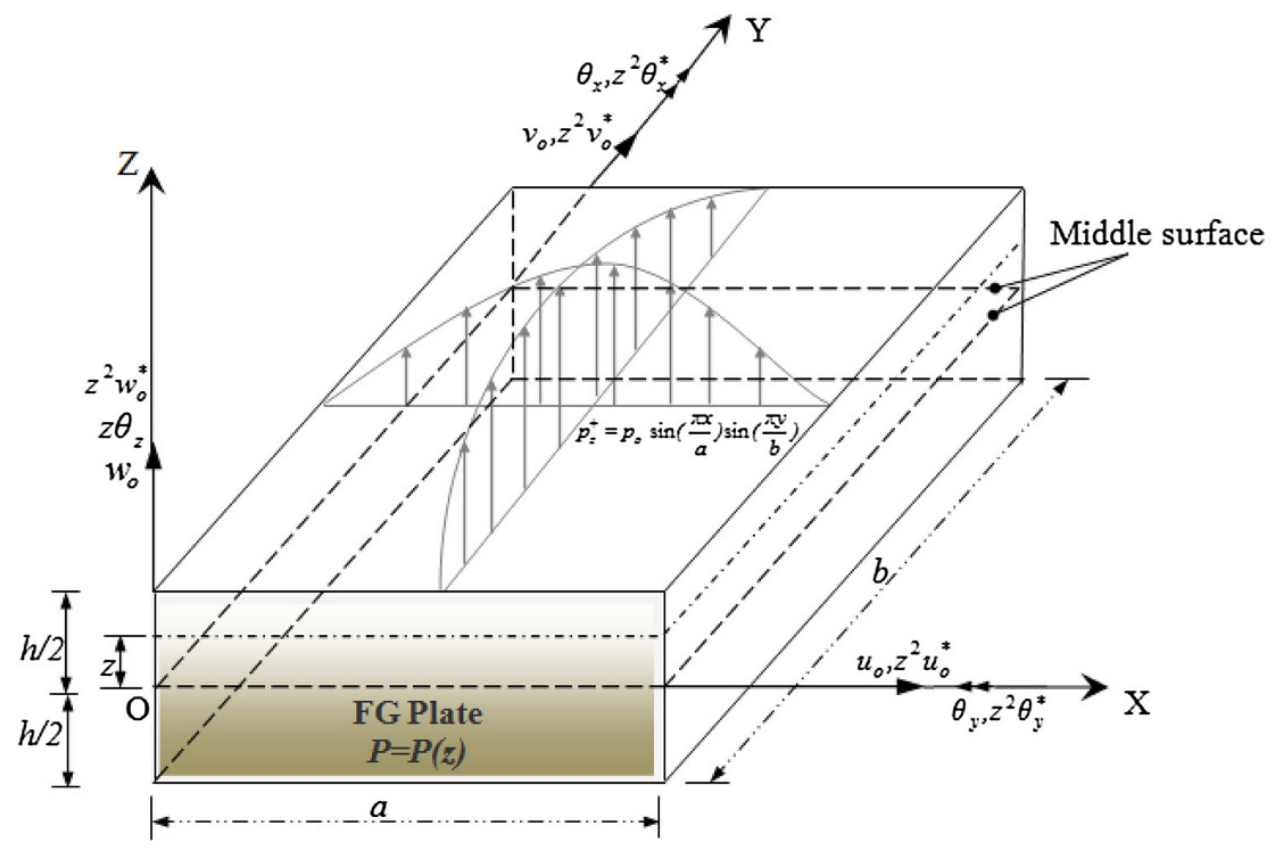

simplify the shear stresses/strains through the plate thickness in an approximate manner, which at times may become unrealistic. These limitations of the FOST necessitate the development of higher order refined theories, in which no such coefficients are required. These refined theories consider the realistic parabolic variation of transverse shear stresses through the plate thickness and warping of the transverse cross section which is definitely essential for the analysis of FG plates.

In the present article, deformation and stress analyses of geometrically thick simply (diaphragm) supported FG plates have been carried out using a higher order shearnormal deformations theory (HOSNT). This HOSNT considers the realistic parabolic distribution of transverse shear strains through the plate thickness. It also considers the effects of both the transverse shear and normal strain/stress and the complete material constitutive relation. Principle of minimisation of potential energy (PMPE) is used to obtain the governing equations of equilibrium for the stress analysis of FG plates. The Navier solution method is used as the solution. The material properties are considered to vary through the thickness according to exponential law distribution. The objectives of the present study is to capture the influence of the higher order terms in the shear deformation theories and the material grading index of FGMs on the static response of FG plates.

\section{Problem definition and material properties}

A linearly elastic transversely loaded square/rectangular simply (diaphragm) supported FG plate of side dimensions $a, b$ and uniform thickness $h$ is considered as shown in Fig. 1. The material properties of the FG plate are assumed to be graded in the thickness direction following the exponential law distribution (Wen et al. 2011) and are expressed as

$C_{i j}^{z}=C_{i j}^{o} e^{\lambda(z+h / 2)} ;$ where, $\lambda=\ln \left(\frac{E_{t}}{E_{b}}\right)$.

Here $C_{i j}^{o}$ indicates material coefficients on the bottom surface. The variation of the mechanical properties versus non-dimensional thickness of plate $z / h$ with different material grading index $\lambda$ is plotted in Fig. 2 .

\section{Displacement field}

The membrane-flexure coupling phenomenon exhibited by FG plate necessitates the use of displacement field containing both, membrane as well as flexural deformation terms which contribute to the overall response of the plate. The displacements $u, v$ and $w$ of a general point $(x, y, z)$ in the plate domain in ' $\mathrm{X}$ ', ' $\mathrm{Y}$ ' and ' $\mathrm{Z}$ ' directions, respectively, are given by

$u(x, y, z)=u_{o}(x, y)+z \theta_{x}(x, y)+z^{2} u_{o}^{*}(x, y)+z^{3} \theta_{x}^{*}(x, y)$

$v(x, y, z)=v_{o}(x, y)+z \theta_{y}(x, y)+z^{2} v_{o}^{*}(x, y)+z^{3} \theta_{y}^{*}(x, y)$

$w(x, y, z)=w_{o}(x, y)+z \theta_{z}(x, y)+z^{2} w_{o}^{*}(x, y)$.

This model is designated as HOSNT11 as it has 11 middle-surface parameters giving rise to non-vanishing 
Fig. 2 Variation of material property with respect to nondimensional thickness of FG plate with different material grading index $\lambda$ (Lambda)
Exponential model of FGM

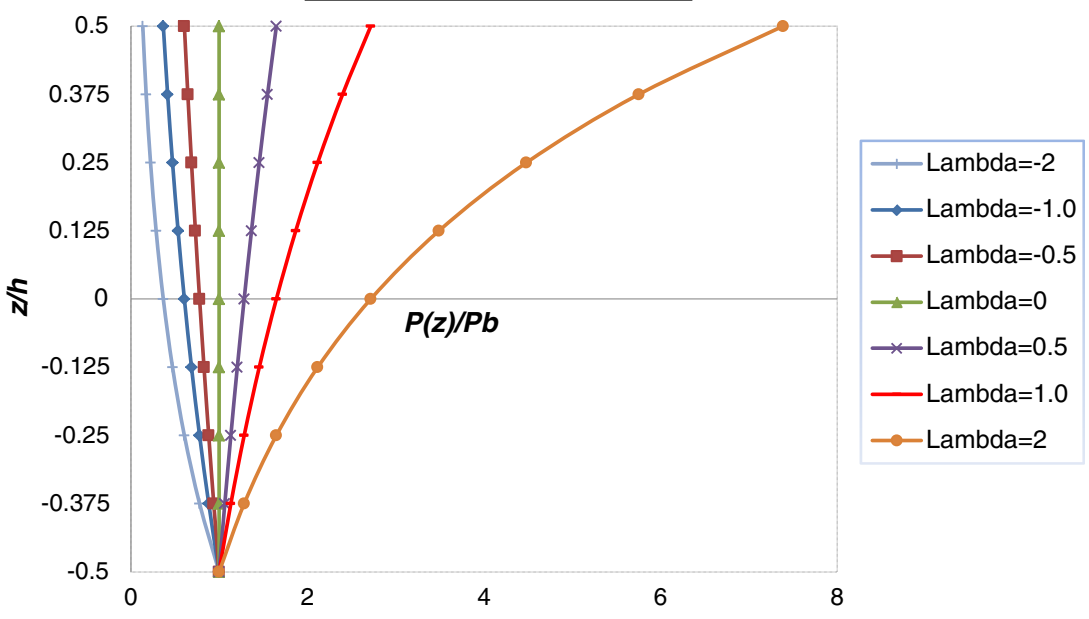

transverse normal strain term varying linearly through the thickness. This theory considers the realistic parabolic distribution of transverse shear strains through the plate thickness. It also considers the effects of both the transverse shear and normal strains/stresses and the complete material constitutive relation. In the above relations, the terms $u, v$ and $w$ are the displacements of a general point ( $x$, $y, z)$ in the laminate domain in the $\mathrm{x}, \mathrm{y}$ and $\mathrm{z}$ directions, respectively. The parameters $u_{o}, v_{o}$ are the in-plane tangential displacements and $w_{o}$ is the transverse displacement of a point $(x, y)$ on the middle surface. The functions $\theta_{x}, \theta_{y}$ are the rotations of the normals to the middle surface about $y$ and $x$ axes, respectively. The parameters $u_{o}^{*}, v_{o}^{*}, w_{o}^{*}, \theta_{x}^{*}, \theta_{y}^{*}$, and $\theta_{z}$ are the higher order terms in the Taylor's series expansion and they represent higher order transverse cross-sectional deformation modes.

\section{Stress-strain relations}

The general linear strain-displacement relation Timoshenko and Goodier (1970) at any point within a plate is considered in the formulation. A FG plate is modelled as an inhomogeneous plate. The material is assumed to be isotropic with varying material properties along plate thickness direction. Generalised Hooke's law for an isotropic material is considered to represent the material constitutive relations. From linear elasticity theory, the generalised Hooke's law for isotropic material can be written as

$\sigma_{i}=C_{i j}(z) \varepsilon_{j} ; i, j=1$ to 6 ,

where $\boldsymbol{\sigma}_{\mathrm{i}}$ is the stress vector, $C_{i j}(z)$ is the stiffness matrix and $\varepsilon_{j}$ is the engineering strain vector of the material at a distance $z$ from the middle surface with reference to the principal material axes $(1,2,3)$. For an isotropic FG plate in a 3D state of stress/strain, the constitutive relations can be written as follows (Reddy 1997):

$$
\left\{\begin{array}{c}
\sigma_{1} \\
\sigma_{2} \\
\sigma_{3} \\
\tau_{12} \\
\tau_{23} \\
\tau_{13}
\end{array}\right\}=\left[\begin{array}{cccccc}
C_{11} & C_{12} & C_{13} & 0 & 0 & 0 \\
C_{12} & C_{22} & C_{23} & 0 & 0 & 0 \\
C_{13} & C_{23} & C_{33} & 0 & 0 & 0 \\
0 & 0 & 0 & C_{44} & 0 & 0 \\
0 & 0 & 0 & 0 & C_{55} & 0 \\
0 & 0 & 0 & 0 & 0 & C_{66}
\end{array}\right]^{z}\left\{\begin{array}{c}
\varepsilon_{1} \\
\varepsilon_{2} \\
\varepsilon_{3} \\
\gamma_{12} \\
\gamma_{23} \\
\gamma_{13}
\end{array}\right\}^{z}
$$

in which

$$
\begin{array}{r}
C_{11}=\frac{(1-v)}{(1+v)(1-2 v)} E(z)=C_{22}=C_{33} \\
C_{12}=\frac{(v)}{(1+v)(1-2 v)} E(z)=C_{13}=C_{23} \\
C_{44}=\frac{1}{2(1+v)} E(z)=C_{55}=C_{66}
\end{array}
$$

Equilibrium equations and natural boundary conditions

PMPE is used to derive the governing equations of equilibrium which is a twenty-second order partial differential equation (PDE) system in terms of eleven middle surface displacement quantities as follows:

$$
\begin{aligned}
& \delta u_{o}: \frac{\partial N_{x}}{\partial x}+\frac{\partial N_{x y}}{\partial y}=0 \\
& \delta v_{o}: \frac{\partial N_{y}}{\partial y}+\frac{\partial N_{x y}}{\partial x}=0 \\
& \delta w_{o}: \frac{\partial Q_{x}}{\partial x}+\frac{\partial Q_{y}}{\partial y}+p_{+}^{z}=0 \\
& \delta \theta_{x}: \frac{\partial M_{x}}{\partial x}+\frac{\partial M_{x y}}{\partial y}-Q_{x}=0 \\
& \delta \theta_{y}: \frac{\partial M_{y}}{\partial y}+\frac{\partial M_{x y}}{\partial x}-Q_{y}=0
\end{aligned}
$$




$$
\begin{aligned}
& \delta \theta_{z}: \frac{\partial S_{x}}{\partial x}+\frac{\partial S_{y}}{\partial y}-N_{z}+\frac{h}{2}\left(p_{+}^{z}\right)=0 \\
& \delta u_{o}^{*}: \frac{\partial N_{x}^{*}}{\partial x}+\frac{\partial N_{x y}^{*}}{\partial y}-2 S_{x}=0 \\
& \delta v_{o}^{*}: \frac{\partial N_{y}^{*}}{\partial y}+\frac{\partial N_{x y}^{*}}{\partial x}-2 S_{y}=0 \\
& \delta w_{o}^{*}: \frac{\partial Q_{x}^{*}}{\partial x}+\frac{\partial Q_{y}^{*}}{\partial y}-2 M_{z}^{*}+\frac{h^{2}}{4}\left(p_{+}^{z}\right)=0 \\
& \delta \theta_{x}^{*}: \frac{\partial M_{x}^{*}}{\partial x}+\frac{\partial M_{x y}^{*}}{\partial y}-3 Q_{x}^{*}=0 \\
& \delta \theta_{y}^{*}: \frac{\partial M_{y}^{*}}{\partial y}+\frac{\partial M_{x y}^{*}}{\partial x}-3 Q_{y}^{*}=0
\end{aligned}
$$

Here, $N_{x}, N_{y}, N_{x y}, N_{z}, M_{x}, M_{y}, M_{x y}, Q_{x}, Q_{y}, S_{x}, S_{y}, N_{x}^{*}$, $N_{y}^{*}, \quad N_{x y}^{*}, \quad M_{x}^{*}, M_{y}^{*}, M_{x y}^{*}, M_{z}^{*}, Q_{x}^{*}, Q_{y}^{*}, S_{x}^{*}, S_{y}^{*}$ represent 'stress resultants', which are the 'stresses' defined in terms of equivalent forces (axial force, shear, or bending moment imposed) acting on the middle surface of plate. The system of equations comprising of equilibrium equations, stress resultant-displacement relations, along with boundary conditions constitutes the complete set of governing equations and also a completely defined and properly posed boundary value problem (BVP).

\section{Solution technique}

Navier solution technique using the double Fourier series is adopted for solving the governing equations of equilibrium for stress analysis of FG plates. The boundary conditions for the simply (diaphragm) supported FG plate using displacement model HOSNT11 defined in Eq. (2a), (2b) and (2c) areAt edges $x=0$ and $x=a$

$$
\begin{array}{r}
v_{o}=0 ; w_{o}=0 ; \theta_{y}=0 ; \theta_{z}=0 ; M_{x}=0 ; N_{x}=0 ; \\
v_{o}^{*}=0 ; w_{o}^{*}=0 ; \theta_{y}^{*}=0 ; M_{x}^{*}=0 ; N_{x}^{*}=0 .
\end{array}
$$

At edges $y=0$ and $y=b$

$$
\begin{array}{r}
u_{o}=0 ; w_{o}=0 ; \theta_{x}=0 ; \theta_{z}=0 ; M_{y}=0 ; N_{y}=0 ; \\
u_{o}^{*}=0 ; w_{o}^{*}=0 ; \theta_{x}^{*}=0 ; M_{y}^{*}=0 ; N_{y}^{*}=0 .
\end{array}
$$

The generalised displacement field for the displacement model 'HOSNT11' to satisfy the above boundary conditions is expanded in double Fourier series as

$$
\left.\begin{array}{rr}
u_{o}=\sum_{m=1}^{\infty} \sum_{n=1}^{\infty} u_{\text {omn }} \cos \alpha_{m} x \sin \beta_{n} y & u_{o}^{*}=\sum_{m=1}^{\infty} \sum_{n=1}^{\infty} u_{o m n}^{*} \cos \alpha_{m} x \sin \beta_{n} y \\
v_{o}=\sum_{m=1}^{\infty} \sum_{n=1}^{\infty} v_{\text {omn }} \sin \alpha_{m} x \cos \beta_{n} y & v_{o}^{*}=\sum_{m=1}^{\infty} \sum_{n=1}^{\infty} v_{o m n}^{*} \sin \alpha_{m} x \cos \beta_{n} y \\
w_{o}=\sum_{m=1}^{\infty} \sum_{n=1}^{\infty} w_{\text {omn }} \sin \alpha_{m} x \sin \beta_{n} y & w_{o}^{*}=\sum_{m=1}^{\infty} \sum_{n=1}^{\infty} w_{o m n}^{*} \sin \alpha_{m} x \sin \beta_{n} y \\
\theta_{x}=\sum_{m=1}^{\infty} \sum_{n=1}^{\infty} \theta_{x m n} \cos \alpha_{m} x \sin \beta_{n} y & \theta_{x}^{*}=\sum_{m=1}^{\infty} \sum_{n=1}^{\infty} \theta_{x m n}^{*} \cos \alpha_{m} x \sin \beta_{n} y \\
\theta_{y}=\sum_{m=1}^{\infty} \sum_{n=1}^{\infty} \theta_{y m n} \sin \alpha_{m} x \cos \beta_{n} y & \theta_{y}^{*}=\sum_{m=1}^{\infty} \sum_{n=1}^{\infty} \theta_{y m n}^{*} \sin \alpha_{m} x \cos \beta_{n} y \\
\theta_{z}=\sum_{m=1}^{\infty} \sum_{n=1}^{\infty} \theta_{z m n} \sin \alpha_{m} x \sin \beta_{n} y
\end{array}\right\}
$$

Transverse loading term is also expanded into a double sine series as

$p_{+}^{z}=\sum_{m=1}^{\infty} \sum_{n=1}^{\infty} p_{+m n}^{z} \sin \alpha_{m} x \sin \beta_{n} y$,

where $\alpha_{m}=\frac{m \pi}{a}$ and $\beta_{n}=\frac{n \pi}{b}$, in which $m, n=$ odd numbers $(1,3,5, \ldots)$.

Using the above generalised displacement field and following the standard steps described in previous section for collecting the coefficients of the 111 displacement degrees of freedom in a $(11 \times 11)$ system of simultaneous equations, the Fourier amplitudes of the displacements are obtained for any fixed values of $m$ and $n$ as follows:

$[\mathrm{X}]_{11 \times 11}\left\{\begin{array}{c}u_{o m n} \\ v_{o m n} \\ w_{\text {omn }} \\ \theta_{x m n} \\ \theta_{y m n} \\ \theta_{z m n} \\ u_{o m n}^{*} \\ v_{o m n}^{*} \\ w_{o m n}^{*} \\ \theta_{x m n}^{*} \\ \theta_{y m n}^{*}\end{array}\right\}_{11 \times 1}=\left\{\begin{array}{c}0 \\ 0 \\ p_{+m n}^{z} \\ 0 \\ 0 \\ \frac{h}{2}\left(p_{+m n}^{z}\right) \\ 0 \\ 0 \\ \frac{h^{2}}{4}\left(p_{+m n}^{z}\right) \\ 0 \\ 0\end{array}\right\}_{11 \times 1}$,

where $[\mathbf{X}]$ is the coefficient matrix using present displacement model (HOSNT11). The elements of the coefficient matrix have been worked out in terms of elements of the sub matrices of plate rigidity matrix for displacement model HOSNT11. The Fourier amplitudes can be easily obtained by solving Eq. (9) with 11 unknown coefficients 
$u_{o_{m n}}, v_{o_{m n}}, w_{o_{m n}}, \theta_{x_{m n}}, \theta_{y_{m n}}, \theta_{z_{m n}}, u_{o_{m n}}^{*}, v_{o_{m n}}^{*}, w_{o_{m n}}^{*}, \theta_{x_{m n}}^{*}$, and $\theta_{y_{m n}}^{*}$. These amplitudes are then used to calculate the generalised displacement components and their derivatives. Further, the generalised displacement components and their derivatives are then used to obtain the stress resultants. These generalised displacement components and their derivatives are back substituted into the strain-displacement relations to obtain the strains. The complete 3D Hooke's law is used to compute in-plane and transverse stresses.

\section{Verification}

We compute the non-dimensional displacements and stresses using displacement model HOSNT11 for isotropic and orthotropic plates mainly because analytical results for the same problem are available for comparison in Wen et al. (2011). The same parameters for validation are available for comparison in Zhang and Zhong (2007) using
3D elasticity approach. The simply (diaphragm) supported square plate problem with isotropic material properties and transversally applied sinusoidal load is considered for validation first. The square plate has a side-length $a=1 \mathrm{~m}$ and thickness-to-side ratio $h / a=0.1$. The elasticity constant, $E$, is taken as unity with Poisson's ratio $v=0.30$. The plate is subjected to a sinusoidal static load $q(x, y)=-q_{0} \sin (\pi x / a) \sin (\pi y / a)$. The normalized load is chosen as $q_{0}=10^{-3} \quad C$, where $C=E \quad(1-v) /$ $[(1+v)(1-2 v)]$. The analytical solutions are obtained for isotropic, orthotropic and FG plates under static loads at the point $(a / 4, a / 4,-h / 4)$. The non-dimensional displacements and stresses for the above-mentioned isotropic plate problem at the same point $(a / 4, a / 4,-h / 4)$ are presented in Table 1 using present higher order displacement model HOSNT11, and compared with the RBF solutions of Wen et al. (2011) and exact 3D elasticity solutions of Zhang and Zhong (2007). For sinusoidal loading, Fourier transverse loading coefficient is considered as $p_{+m n}^{z}=q_{0} / m n$, and $m$, $n=1$. As can be seen from the presented results,

Table 1 Displacements and stresses at $(a / 4, a / 4,-h / 4)$ in an isotropic square plate $(h / a=0.1)$ subjected to transverse sinusoidal load

\begin{tabular}{|c|c|c|c|c|c|c|c|}
\hline \multirow[t]{2}{*}{ Theory } & \multirow[t]{2}{*}{ Point $(x, y, z)$} & \multicolumn{6}{|c|}{ Non-dimensional displacements and stresses } \\
\hline & & $u / a\left(\times 10^{-3}\right)$ & $v / a\left(\times 10^{-3}\right)$ & $w / h\left(\times 10^{-1}\right)$ & $\sigma_{x} / C\left(\times 10^{-3}\right)$ & $\tau_{x z} / C\left(\times 10^{-3}\right)$ & $\tau_{x y} / C\left(\times 10^{-3}\right)$ \\
\hline Exact $^{\mathrm{a}}$ (3D elasticity) & $(a / 4, a / 4,-h / 4)$ & -1.4796 & -1.4796 & -1.9750 & 4.8997 & -0.8955 & -2.6561 \\
\hline \multirow[t]{2}{*}{$\mathrm{RBF}^{\mathrm{b}} 3 \mathrm{D}$ analysis } & $(a / 4, a / 4,-h / 4)$ & -1.4796 & -1.4796 & -1.9750 & 4.8997 & -0.8955 & -2.6561 \\
\hline & (\% Error) & 0.000 & 0.000 & 0.000 & 0.000 & 0.000 & 0.000 \\
\hline \multirow[t]{2}{*}{ HOSNT11 } & $(a / 4, a / 4,-h / 4)$ & -1.4797 & -1.4797 & -1.9751 & 4.8782 & -0.8948 & -2.6562 \\
\hline & (\% Error) & 0.007 & 0.007 & 0.005 & -0.439 & -0.078 & 0.004 \\
\hline \multirow[t]{2}{*}{ HOSNT $11^{c}$} & $(a / 4, a / 4,-h / 4)$ & -1.4745 & -1.4745 & -1.9957 & 4.8876 & -0.8948 & -2.6469 \\
\hline & (\% Error) & -0.345 & 1.048 & -0.247 & -0.075 & -0.346 & -0.345 \\
\hline
\end{tabular}

a Taken from Zhang and Zhong (2007)

b Taken from Wen et al. (2011)

c The transverse load is considered to be applied at the middle surface $(z=0)$ of the plate in the formulation

Table 2 Displacements and stresses at $(a / 4, a / 4,-h / 4)$ for simply (diaphragm) supported square orthotropic plate $(h / a=0.1)$ subjected to transverse sinusoidal load

\begin{tabular}{|c|c|c|c|c|c|c|c|}
\hline \multirow[t]{2}{*}{ Theory } & \multirow[t]{2}{*}{ Point $(x, y, z)$} & \multicolumn{6}{|c|}{ Non-dimensional displacements and stresses } \\
\hline & & $u / a\left(\times 10^{-3}\right)$ & $v / a\left(\times 10^{-3}\right)$ & $w / h\left(\times 10^{-1}\right)$ & $\sigma_{x} / C_{33}\left(\times 10^{-3}\right)$ & $\tau_{x z} / C_{33}\left(\times 10^{-3}\right)$ & $\tau_{x y} / C_{33}\left(\times 10^{-3}\right)$ \\
\hline Exact $^{\mathrm{a}}$ (3D elasticity) & $(a / 4, a / 4,-h / 4)$ & -1.0766 & -1.1178 & -1.4840 & 6.7650 & -1.0670 & -2.2020 \\
\hline \multirow[t]{2}{*}{$\mathrm{RBF}^{\mathrm{b}} 3 \mathrm{D}$ analysis } & $(a / 4, a / 4,-h / 4)$ & -1.0766 & -1.1178 & -1.4842 & 6.7655 & -1.0664 & -2.2017 \\
\hline & (\% Error) & 0.000 & 0.000 & 0.013 & 0.007 & -0.056 & -0.014 \\
\hline \multirow[t]{2}{*}{ HOSNT11 } & $(a / 4, a / 4,-h / 4)$ & -1.0766 & -1.1178 & -1.4842 & 6.7469 & -1.0651 & -2.2017 \\
\hline & (\% Error) & 0.000 & 0.000 & 0.013 & -0.268 & -0.178 & -0.014 \\
\hline \multirow[t]{2}{*}{ HOSNT11 ${ }^{\mathrm{c}}$} & $(a / 4, a / 4,-h / 4)$ & -1.0765 & -1.1093 & -1.4982 & 6.7651 & -1.0664 & -2.1931 \\
\hline & (\% Error) & -0.009 & -0.760 & 0.957 & 0.001 & -0.056 & -0.404 \\
\hline
\end{tabular}

\footnotetext{
a Taken from Zhang and Zhong (2007)

b Taken from Wen et al. (2011)

c The transverse load is considered to be applied at the middle surface $(z=0)$ of the plate in the formulation
} 
HOSNT11 computes very accurate results with $0.007 \%$ errors in the in-plane displacements and $0.005 \%$ error in transverse deflection for isotropic plates. Errors (\%) in the stresses are $-0.439,-0.078$ and 0.004 in $\sigma_{x}, \tau_{x z}$ and $\tau_{x y}$, respectively.

A sinusoidally loaded, simply (diaphragm) supported orthotropic square plate problem is considered next (Wen et al. 2011). The square plate has a side-length $a=1 \mathrm{~m}$ and thicknesses-to-side ratio, $h / a=0.1$. Transverse load is defined as $q(x, y)=-q_{0} \sin (\pi x / a) \sin (\pi y / a)$. The normalized load is chosen here as $q_{0}=10^{-3} C_{33}$, where $C_{33}=$ $E_{3}\left[\left(1-v_{12} v_{21}\right) /\left\{1-v_{12}^{2}-2 v_{12} v_{21}\left(1+v_{12}\right)\right\}\right]$. The orthotropic material properties for the numerical evaluation are $E_{2} / E_{1}=0.5, E_{3}=E_{2}, v_{12}=0.3, v_{21}=0.15, G_{12}=G_{23}=$ $G_{13}=E_{2} / 2\left(1+v_{12}\right)$. Other material properties have been derived on the basis of law of symmetry of orthotropic materials. The non-dimensional displacements and stresses at the point $(a / 4, a / 4,-h / 4)$ are presented based on present higher order model HOSNT11, and compared with the RBF solutions of Wen et al. (2011) and exact 3D elasticity solutions by Zhang and Zhong (2007). The comparisons of various solutions are presented in Table 2. It is clearly seen from the presented results that HOSNT11 computes the most accurate results with zero errors in the in-plane displacements and $0.013 \%$ error in transverse deflection for orthotropic plates. Errors $(\%)$ in the stresses are $-0.268,-0.178$ and -0.014 in $\sigma_{x}, \tau_{x z}$ and $\tau_{x y}$, respectively.

\section{Numerical examples}

Example-1: A FG plate subjected to sinusoidal load

A sinusoidally loaded, simply (diaphragm) supported, square FG plate of side $a=1 \mathrm{~m}$ and thicknesses-to-width ratio, $h / a=0.1$ is considered. The elastic modulii are $E_{t}=70 \mathrm{GPa}, E_{b}=151 \mathrm{GPa}$, and the Poisson's ratio is $v=0.3$. Here $E_{t}$ and $E_{b}$ indicate Young's modulii on the top (i.e., $z=+h / 2$ ) and bottom (i.e., $z=-h / 2$ ) surfaces of the FG plate, respectively. Sinusoidal static load is assumed as $q(x, y)=-q_{0} \sin (\pi x / a) \sin (\pi y / a)$. The normalized load is chosen as $q_{0}=10^{-3} C_{33}^{o}$, where $C_{33}^{o}=$ $E_{b}(1-v) /[(1+v)(1-2 v)]$. The analytical solutions for deflections and stresses of the above-described graded material plate are presented at the point $(a / 4, a / 4,-h / 4)$ by Wen et al. (2011) based on the state-space approach in the Laplace transform domain, transforming it to a onedimensional problem, and solved using RBF. The exact 3D elasticity solutions of this problem are presented by Zhang and Zhong (2007). The non-dimensional displacements and stresses are evaluated at the same point $(a / 4, a / 4,-h / 4)$ using present model (HOSNT11) and presented in Table 3 along with the analytical solutions by Wen et al. (2011) and 
Fig. 3 Through thickness variations of in-plane displacement of simply (diaphragm) supported FG square plates $(h / a=0.1)$ under sinusoidal load

Fig. 4 Through thickness variations of transverse displacement of simply (diaphragm) supported FG square plates $(h / a=0.1)$ under sinusoidal load

Fig. 5 Through thickness variations of in-plane normal stress of simply (diaphragm) supported FG square plates $(h / a=0.1)$ under sinusoidal load
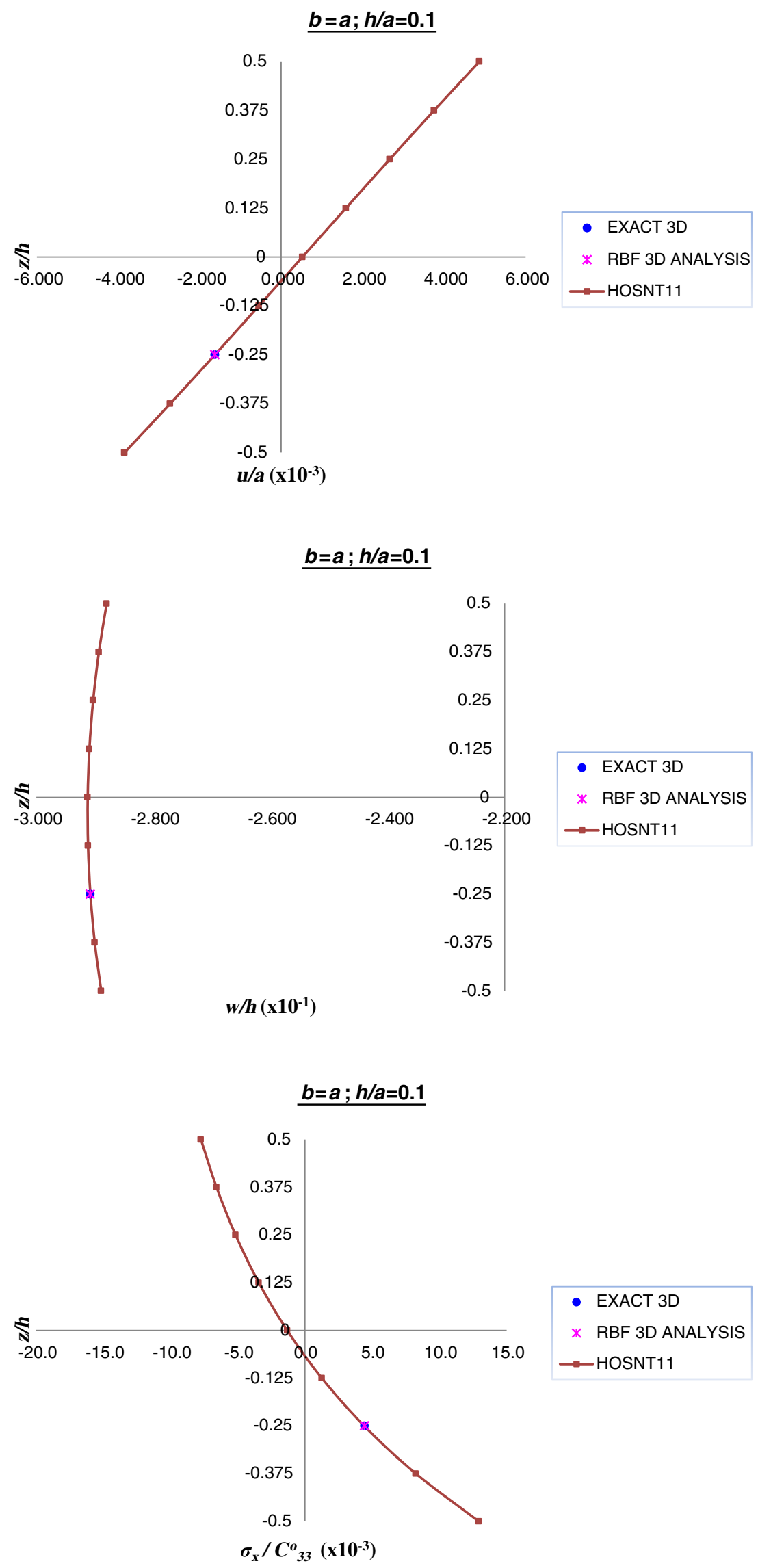
Fig. 6 Through thickness variations of transverse shear stress of simply (diaphragm) supported FG square plates $(h / a=0.1)$ under sinusoidal load
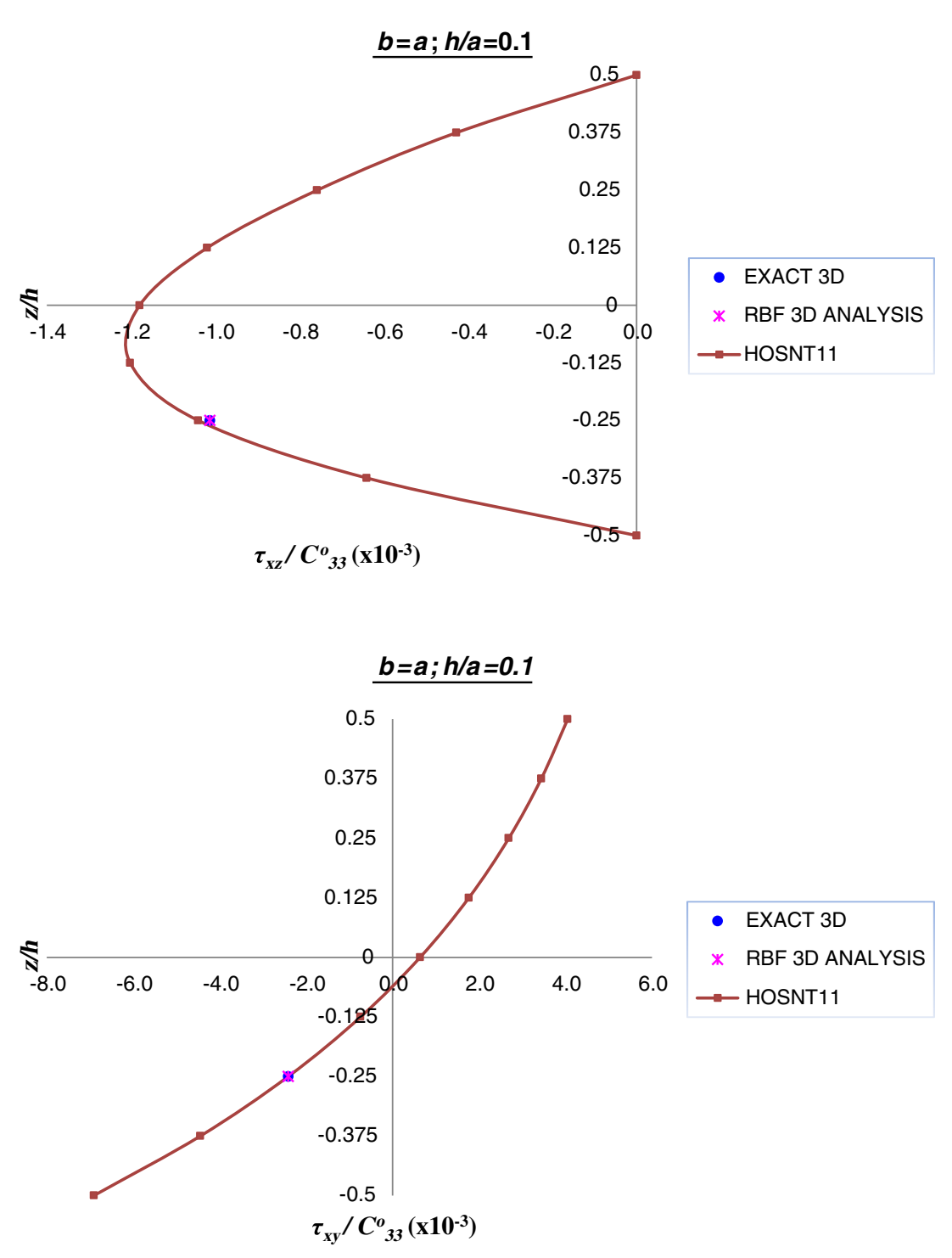

Fig. 7 Through thickness variations of in-plane shear stress of simply (diaphragm) supported FG square plates $(h / a=0.1)$ under sinusoidal load the exact 3D elasticity solutions by Zhang and Zhong (2007). The FOST and CPT solutions are also worked out independently and tabulated along with the HOSNT11 solutions for comparison shake. The through thickness variation of displacements and stresses for square FG plates $(h / a=0.1)$ are plotted in Figs. 3, 4, 5, 6, 7 .

The results obtained using model HOSNT11 are is in close agreement with the 3D elasticity solutions (Zhang and Zhong 2007) and also to the RBF solutions (Wen et al. 2011) with highest number of collocation points. HOSNT11 has an error of $0.006 \%$ in the in-plane displacements and $0.007 \%$ error in transverse deflection. The deflections and stresses at point $(a / 4, a / 4,-h / 4)$ using HOSNT11 are also presented in Table 3 for geometrically thick plates of thickness ratios ( $h / a) 0.2$ and 0.5 for future references.

Here, it is interesting to note that the neutral surface of the FG plate does not coincide with the middle surface $(z=0)$ of the plate, and it is found to be at $z=-0.06 h$, $-0.05 h$ and $-0.04 h$ for the FG plates of thickness ratios $(h /$ a) $0.1,0.2$ and 0.25 , respectively using the most accurate HOSNT11 model. This shift of the neutral surface occurs towards the stiffer material side. It can be very clearly pointed out from the presented results that the higher order transverse normal deformation terms in choosing the displacement field cannot be neglected in the formulation for the thick FG plates, especially when the thickness ratios $(h / a)$ equals 0.2 or is more than 0.2 . This highly accurate $2 \mathrm{D}$ formulation can be used to capture the behaviour of transversely loaded FG plates more closely, especially for thicker plates $(h / a \geq 0.1)$, and it is totally justified and recommended when the thickness-to-side ratio $(\mathrm{h} / \mathrm{a})$ exceeds 0.2. Using this higher order displacement formulation, the transverse loads can be applied at any surface of the plate. 
Table 4 Maximum deflections and stresses in a simply (diaphragm) supported FG square plate ( $h / a=0.1)$ subjected to uniform load

\begin{tabular}{|c|c|c|c|c|c|c|c|}
\hline \multirow[t]{2}{*}{ Theory } & \multicolumn{7}{|c|}{ Non-dimensional displacements and stresses } \\
\hline & $\begin{array}{l}\text { Point } \\
(x, y, z)\end{array}$ & $\begin{array}{l}u / a\left(\times 10^{-3}\right) \\
(x=0, y=a / 2)\end{array}$ & $\begin{array}{l}v / a\left(\times 10^{-3}\right) \\
(x=a / 2, y=0)\end{array}$ & $\begin{array}{l}w / h\left(\times 10^{-1}\right) \\
(x=a / 2, y=a / 2)\end{array}$ & $\begin{array}{l}\sigma_{x} / C_{33}^{o}\left(\times 10^{-3}\right) \\
(x=a / 2, y=a / 2)\end{array}$ & $\begin{array}{l}\tau_{x z} / C_{33}^{o}\left(\times 10^{-3}\right) \\
(x=0, y=a / 2)\end{array}$ & $\begin{array}{l}\tau_{x y} / C_{33}^{o}\left(\times 10^{-3}\right) \\
(x=0, y=0)\end{array}$ \\
\hline \multirow[t]{9}{*}{ HOSNT11 } & $z=h / 2$ & 16.246 & 16.246 & -9.087 & -22.416 & 0 & 14.750 \\
\hline & $z=3 h / 8$ & 12.499 & 12.499 & -9.127 & -19.092 & -1.848 & 12.286 \\
\hline & $z=h / 4$ & 8.849 & 8.849 & -9.157 & -14.981 & -3.156 & 9.428 \\
\hline & $z=h / 8$ & 5.264 & 5.264 & -9.176 & -9.927 & -4.187 & 6.063 \\
\hline & $z=0$ & 1.709 & 1.709 & -9.186 & -3.748 & -4.817 & 2.039 \\
\hline & $z=-h / 8$ & -1.848 & -1.848 & -9.185 & 3.777 & -4.891 & -2.834 \\
\hline & $z=-h / 4$ & -5.443 & -5.443 & -9.173 & 12.913 & -4.224 & -8.802 \\
\hline & $z=-3 h / 8$ & -9.107 & -9.107 & -9.152 & 23.970 & -2.589 & -16.174 \\
\hline & $z=-h / 2$ & -12.875 & -12.875 & -9.120 & 37.320 & 0 & -25.334 \\
\hline \multirow[t]{9}{*}{ HOSNT $11^{\text {a }}$} & $z=h / 2$ & 16.606 & 16.606 & -9.186 & -22.217 & 0 & 15.228 \\
\hline & $z=3 h / 8$ & 12.821 & 12.821 & -9.229 & -18.925 & -1.756 & 12.864 \\
\hline & $z=h / 4$ & 9.124 & 9.124 & -9.261 & -14.850 & -3.106 & 10.038 \\
\hline & $z=h / 8$ & 5.484 & 5.484 & -9.283 & -9.836 & -4.171 & 6.633 \\
\hline & $z=0$ & 1.870 & 1.870 & -9.293 & -3.695 & -4.827 & 2.502 \\
\hline & $z=-h / 8$ & -1.751 & -1.751 & -9.293 & 3.798 & -4.924 & -2.542 \\
\hline & $z=-h / 4$ & -5.410 & -5.410 & -9.283 & 12.914 & -4.277 & -8.731 \\
\hline & $z=-3 h / 8$ & -9.138 & -9.138 & -9.261 & 23.974 & -2.666 & -16.353 \\
\hline & $z=-h / 2$ & -12.969 & -12.969 & -9.229 & 37.362 & 0 & -25.758 \\
\hline
\end{tabular}

a The transverse load is considered to be applied at the middle surface $(z=0)$ of the plate in the formulation

Example-2: A FG square plate subjected to uniform transverse load

The FG square plate $(h / a=0.1)$ problem described above is analysed here under uniform load $q_{0}$. The similar material property as described in Example-1 is used for the numerical evaluation. For the case of uniformly distributed load, Fourier transverse loading coefficient is considered as $p_{+m n}^{z}=16 q_{0} / \pi^{2} m n$; for odd integers $m, n=1,3,5, \ldots$. The non-dimensional maximum displacements and stresses of the FG square plate are presented along the thickness using HOSNT11 in Table 4. It is evident from the results presented that the neutral surface of the FG plate does not lie in the middle surface $(z=0)$ of plate. For the considered material property and gradation coefficient of FG plate, the neutral surface here also is shifted at $z=-0.06 h$. The shift of the neutral surface of the plate from the middle surface is towards the stiffer material zone and independent of the magnitude or type of loading. It only depends on the material gradation coefficient along the thickness.

\section{Concluding remarks}

In this article, a 2D plate theory for the deformation and stress analyses of moderately thick functionally graded elastic, rectangular/square plates is presented using higher order shear and normal deformation theory (HOSNT11). The material properties of FG plates are assumed to vary in the thickness direction according to an exponential law distribution. The governing equations of equilibrium are obtained using the PMPE. Navier solution technique employing double Fourier series is used to get the results with desired level of accuracy for simply (diaphragm) supported FG plates. For all cases studied, the computed displacement and stress parameters are found to be close to the exact analytical solutions. These numerical solutions with high accuracy can be used as benchmark to assess any other analytical/computational models for FG plates. Although the presented formulation for FG thick plates using HOSNT11 involves large computations compared to FOST and CPT, the accuracy of the obtained numerical results is as good as the exact 3D elasticity solutions. This particular higher order model (HOSNT11) yields the most accurate results for the transversely loaded simply (diaphragm) supported FG plates. The benefit of this approach is that a $2 \mathrm{D}$ theory is able to predict solutions close to 3D elasticity solutions. The location of neutral surface of the FG plates depends upon the gradation index of FGMs and also its thickness ratio. The shift of the neutral surface from the middle surface of the FG plates is towards the stiffer material zone. It does not 
depend upon the aspect ratio of the plate and the external loads applied.

Open Access This article is distributed under the terms of the Creative Commons Attribution License which permits any use, distribution, and reproduction in any medium, provided the original author(s) and the source are credited.

\section{References}

Birman V, Byrd LW (2007) Modeling and analysis of functionally graded materials and structures. ASME Appl Mech Rev 60:195-216

Ferreira AJM, Batra RC, Roque CMC, Qian LF, Martins PALS (2005) Static analysis of functionally graded plates using thirdorder shear deformation theory and a meshless method. Compos Struct 69:449-457

Ferreira AJM, Roque CMC, Jorge RMN, Fasshauer GE, Batra RC (2007) Analysis of functionally graded plates by a robust meshless method. Mech Adv Mater Struct 14:577-587

Jha DK, Kant T, Singh RK (2013) A critical review of recent research on functionally graded plates. Compos Struct 96:833-849

Kant T (1982) Numerical analysis of thick plates. Comp Meth App Mech Eng 31:1-18

Kant T, Manjunatha BS (1994) On accurate estimation of transverse stresses in multilayer laminates. Comput Struct 50(3):351-365

Kant T, Swaminathan K (2002) Analytical solutions for the static analysis of laminated composite and sandwich plates based on a higher-order refined theory. Compos Struct 56:329-344

Koizumi M (1997) FGM activities in Japan. Compos B 28:1-4

Mian AM, Spencer AJM (1998) Exact solutions for functionally graded and laminated elastic materials. J Mech Phys Solids 46(12):2283-2295
Nguyen TK, Sab K, Bonnet G (2007) Shear correction factors for functionally graded plates. Mech Adv Mater Struct 14(8):567-575

Nguyen TK, Sab K, Bonnet G (2008) First-order shear deformation plate models for functionally graded materials. Compos Struct $83: 25-36$

Pagano NJ (1969) Exact solutions for composite laminates in cylindrical bending. J Compos Mater 3(3):398-411

Pagano NJ (1970) Exact solutions for rectangular bidirectional composites and sandwich plates. J Compos Mater 4(1):20-34

Pandya BN, Kant T (1988) Higher-order shear deformable theories for flexure of sandwich plates-finite element evaluations. Int $\mathbf{J}$ Solids Struct 24(12):1267-1286

Reddy JN (1997) Mechanics of laminated composite plates, theory and analysis, 1st edn. CRC Press, New York

Srinivas S, Rao AK (1970) Bending, vibration and buckling of simply supported thick orthotropic plates and laminates. Int J Solids Struct 6:1463-1481

Srinivas S, Joga Rao CV, Rao AK (1970) An exact analysis for vibration of simply supported homogeneous and laminated thick rectangular plates. J Sound Vib 12:187-199

Suresh S, Mortensen A (1998) Fundamentals of functionally graded materials, 1st edn. IOM Communications, London

Timoshenko SP, Goodier JN (1970) Theory of elasticity, 3rd international edn, McGraw-Hill, Singapore

Vel SS, Batra RC (2002) Exact solutions for thermoelastic deformations of functionally graded thick rectangular plates. AIAA J 40(7):1421-1433

Wen PH, Sladek J, Sladek V (2011) Three-dimensional analysis of functionally graded plates. Int $J$ Numer Meth Eng 87(10):923-942

Zhang C, Zhong Z (2007) Three-dimensional analysis of a simply supported functionally graded plate based on Haar wavelet method. Acta Mech Solida Sin 28(3):217-223 